\title{
Extended isogeometric analysis using analysis-suitable T-splines for plane crack problems
}

\author{
S. H. Habib*, I. Belaidi** \\ *Department of Mechanical Engineering, University of M'hamed Bougara, Boumerdes, Algeria, \\ E-mail: hb_houcine@yahoo.fr \\ **Department of Mechanical Engineering, University of M'hamed Bougara, Boumerdes, Algeria, \\ E-mail: idir.belaidi@gmail.com \\ cross $^{\text {ref }}$ http://dx.doi.org/10.5755/j01.mech.23.1.13475
}

\section{Introduction}

The brutal fracture problem has a great importance in some industrial fields, such as in aeronautics, aerospace and nuclear. This phenomenon has very serious consequences, lead to the need for analysing more and better understanding the mechanical behaviour of structures, with taking into account the effects of the fort and the weak discontinuities, especially in critical conditions. It is a scientific challenge that represents an important issue, analytically as well as numerically.

The complexity of the analytical solutions even for simple cases imposes to setting up effective numerical methods to model the mechanical behaviour. Various studies have been developed using different methods, such as extended finite element method (XFEM) [1], boundary element method (BEM) [2], element free Galerkin method (EFGM) [3], and other methods [4, 5]. Recently, a large field was opened by Hughes et al. [6] offers the possibility of exploiting computer aided design (CAD) tools in the analysis methods using isoparametric concept. This novel alternative method called isogeometric analysis (IGA). The basic idea of this method is use the computational geometry technologies as shape bases to describe exactly the geometry and also to approximate the unknown fields. Following this discovery, several researches in various fields have been investigated by this method, including: structural dynamics [7], composite materials [8], fluidstructure interaction [9], electromagnetic problems [10], contact problems [11], turbulent flow [12], aero-dynamics [13] and thermomechanical problems [14]. However, in fracture mechanics problems, Benson et al. [15] and De Luycker et al. [16] have proposed extended isogeometric analysis (XIGA) for modelling cracks. In this method the general principle of the XFEM is used into IGA by including the asymptotic and signed distance enrichment functions. Therefore this method has the advantages of both XFEM and IGA, which are summarized by the ability to represent complex geometries independently of any discontinuities and without explicit meshing for obtain solution with higher orders.

There are many CAD basis functions can be used in isogeometric analysis, the most widely used of them are Non Uniform Rational B-splines (NURBS) due to their properties like continuity, smoothness, variation diminishing, convex hull and possibility of using Knot insertion and degree elevation refinements. They have the ability to describe exactly all conic sections but they are not well for all complex geometries, even for multiple patches NURBS generate a complicated mesh of control points and this leads to produce superfluous control points. In order to handle these disadvantages Sederberg et al. [17] proposed T-splines as a generalized tool of NURBS, in which the index space (T-mesh) cans have T-junctions and locally refined [18]. Therefore the major advantages of this technique are local refinement and ability to represent complex geometries with minimal number of control points compared with those used in NURBS.

According to their ability in engineering design, $\mathrm{T}$-splines have been used by the analysts to serves as basis functions for isogeometric analysis in many advanced searches. However T-splines bases are not always valid to use in analysis for different geometric configurations, because there are not rules ensure these bases to be linear independent and form a partition of unity. Li et al. [19] introduced analysis-suitable T-splines, wherein for any choice of knot vectors the blending functions are linearly independent. Like NURBS bases, analysis-suitable Tspline bases have the important properties of the analysis basis functions. Moreover, they provide an efficient algorithm which allows making highly localized refinement [20].

In this paper, plane crack problem is analysed numerically using XIGA, which is enhanced by adopting analysis-suitable $\mathrm{T}$-splines in order to approximate the solution, construct the geometry and make the local refinement around the discontinuities. The asymptotic functions are used to model the crack tip, and the M-integral is used to evaluate the stress intensity factors.

\section{Analysis-suitable T-splines}

Analysis-suitable T-splines are a subset of $\mathrm{T}$ splines which have a restricted T-mesh topology; it is essentially based on the positions of the T-junctions in the Tmesh. And that by representation extensions in all $\mathrm{T}$ junctions, if there are intersections between $\mathrm{T}$-junction extensions, then the extended T-mesh is not analysis suitable T-splines. A T-junction extension is composed by extension of two line segments, the first is from the Tjunction until one edge or vertex in the opposite direction of a missing edge, and the second is from the T-junction until two edges or vertices in the other direction, an example is shown in Fig. 1.

\subsection{Building an analysis-suitable T-spline}

For a given analysis-suitable T-mesh and net of 
control points $\left(P_{\alpha}\right)$, an analysis-suitable T-spline surface is given by:

$$
S=\sum_{\alpha=1}^{k} R_{\alpha} \mathrm{P}_{\alpha}
$$

where $R_{\alpha}$ is the blending function of the local knot vectors.

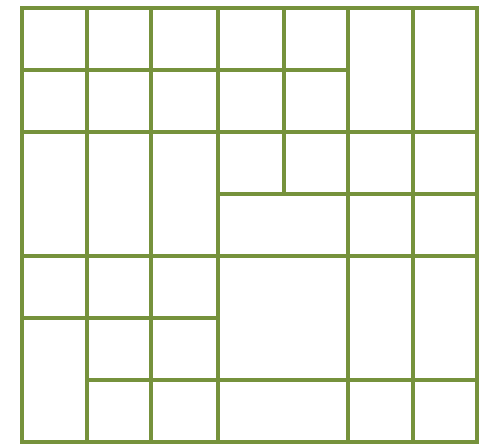

a

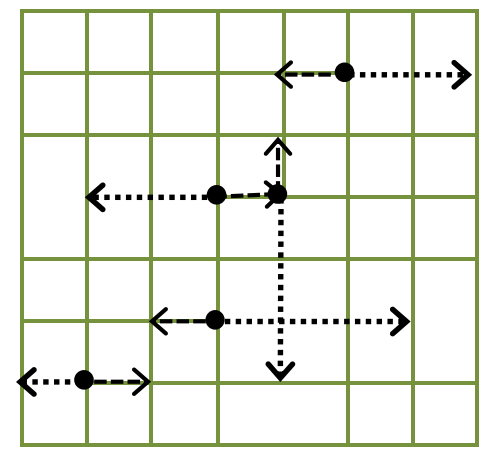

b

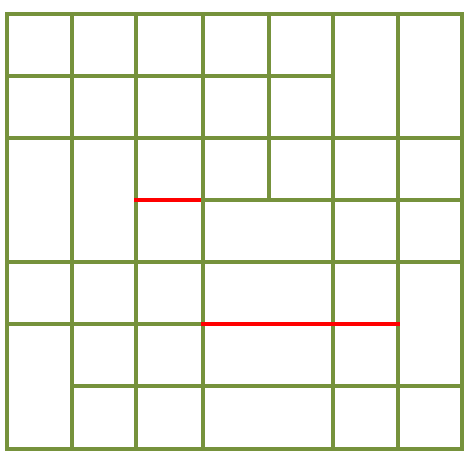

Fig. 1 a - T-mesh; b - extended T-mesh (black points are Tjunctions); c - Analysis-suitable T-mesh (formed by adding the red edges)

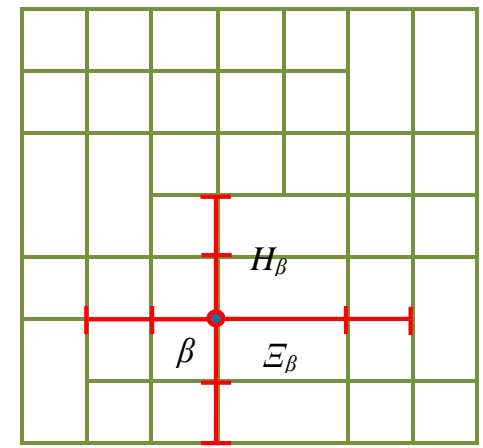

Fig. 2 Local knot vectors of one anchor in the case of bicubic orders
$\Xi_{\alpha}$ and $H_{\alpha}$ which correspond to the anchor $\alpha$ (e.g., Fig. 2 shows local knot vectors of the anchor $\beta$ ), her formula is written in terms of B-spline bases $N_{i, p}$ and $M_{j, q}$ as:

$$
R_{\alpha}(\xi, \eta)=\frac{w_{\alpha} B_{\alpha}(\xi, \eta)}{\sum_{\lambda=1}^{k} w_{\lambda} B_{\lambda}(\xi, \eta)}, \quad B_{\alpha}=N_{i, p}(\xi) M_{j, q}(\eta),
$$

$k$ is the number of the anchors in T-mesh, $w_{\alpha}$ is a set of control point weights, $p$ and $q$ are orders of the basis functions corresponding to the parametric directions $\xi$ and $\eta$, respectively.

The B-spline basis functions in one parametric direction are given by

$$
N_{i, 0}(\xi)=\left\{\begin{array}{cc}
1 & \text { if } \xi_{i} \leq \xi<\xi_{n+p+1} \\
0 & \text { otherwise }
\end{array} \text { for } p=0,\right.
$$

and

$$
\begin{aligned}
& N_{i, p}(\xi)=\frac{\xi-\xi_{i}}{\xi_{i+p}-\xi_{i}} N_{i, p-1}(\xi)+ \\
& +\frac{\xi_{i+p+1}-\xi}{\xi_{i+p+1}-\xi_{i+1}} N_{i+1, p-1}(\xi) \text { for } p>0 .
\end{aligned}
$$

\subsection{Local refinement of analysis-suitable T-splines}

In order to make a local refinement in T-splines, knots must be inserted to selected local knot vectors, so some basis functions are subdivided [18]. For example, in the case of cubic orders (as in the present study) and one inserted knot $\mathrm{z}$ to a local vector, two basis functions are produced. We can combine them linearly to form the original basis function as:

$$
N\left(\xi \mid \xi_{1}, \ldots, \xi_{5}\right)=X N\left(\xi \mid \xi_{1}, \ldots, \xi_{4}\right)+Y N\left(\xi \mid \xi_{2}, \ldots, \xi_{5}\right)
$$

where

$$
X=\left\{\begin{array}{cc}
\frac{z-\xi_{1}}{\xi_{4}-\xi_{1}} & \text { for } \mathrm{z}<\xi_{4} ; \\
1 & \text { for } \mathrm{z} \geq \xi_{4}
\end{array}\right.
$$

and

$$
Y=\left\{\begin{array}{cc}
\frac{\xi_{5}-\mathrm{z}}{\xi_{5}-\xi_{2}} & \text { for } \mathrm{z}>\xi_{2} ; \\
1 & \text { for } \mathrm{z} \leq \xi_{2} .
\end{array}\right.
$$

For more than one inserted knot, the application of these equations is repeated until derive all coefficients.

The original basis functions $B^{1}$ and the generated basis functions $B^{2}$ of a T-spline space can be combined using the refinement operator $\boldsymbol{M}$ in linear system as:

$$
N_{1}=M N_{2},
$$

where $N_{1}$ and $N_{2}$ are the column vectors of basis functions of the original and the refined $\mathrm{T}$-spline space, respectively.

A special algorithm of refinement was introduced by Scott et al. [20] in order to make the local refinement of analysis-suitable T-spline spaces. It consists of the follow- 
ing steps:

- Create the refined T-mesh $T_{2}$ from the original analysis-suitable T-mesh $T_{s 1}$.

- $\quad$ Form the extended T-mesh of $T_{2}$.

- If the extended T-mesh of $T_{2}$ has intersecting Tjunction extensions, one edge must inserted into $T_{2}$ in such a way that reduce the number of the intersections.

- $\quad$ Repeat step 3 until the extended T-mesh has no Tjunction extensions intersect.

- $\quad$ Compute the refinement matrix $\boldsymbol{M}$. For more detail refer you to [20].

\section{Extended isogeometric analysis (XIGA)}

\subsection{Governing equation}

Consider a cracked body $\Omega$ with outer boundary $\Gamma$ subjected to a uniform body forces $f^{b}$, traction forces $f^{t}$ applied at $\Gamma_{t}$ and displacement conditions applied at $\Gamma_{u}$, in the state of equilibrium. The crack boundary $\Gamma_{c}$ is considered to be traction free Fig. 3 .

The strong form of the equilibrium equation and the boundary conditions are defined as [1]:

$$
\begin{aligned}
& \nabla \sigma+\boldsymbol{f}^{b}=0 \text { in } \Omega ; \\
& \sigma \boldsymbol{n}=\boldsymbol{f}^{t} \text { on } \Gamma_{t} ; \\
& \sigma \boldsymbol{n}=0 \text { on } \Gamma_{c} ; \\
& u=\bar{u} \text { on } \Gamma_{u} .
\end{aligned}
$$

The variational formulation of the boundary value problem is defined as:

$$
\int_{\Omega} \sigma \delta \varepsilon d \Omega=\int_{\Omega} f^{b} \delta u d \Omega+\int_{\Gamma_{t}} f^{t} \delta u d \Gamma
$$

where $\sigma$ is the stress tensor, $\varepsilon$ is the strain tensor and $\boldsymbol{n}$ is the unit outward normal.

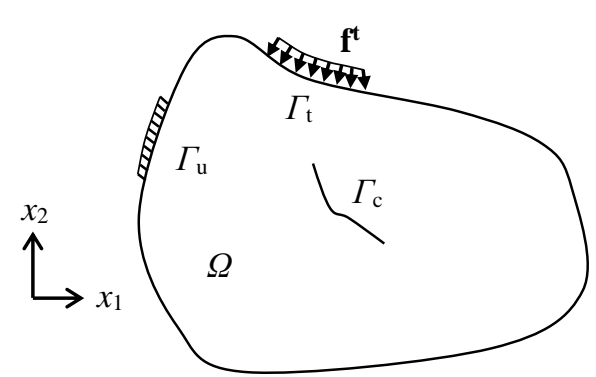

Fig. 3 An arbitrary cracked body with boundary conditions

\subsection{Discretization}

The Extended isogeometric analysis (XIGA) $[15,16]$ uses the same methodology of the extended finite element method (XFEM) for modelling the discontinuities but with basis functions derived from the geometry like in isogeometric analysis [6]. For the crack problems, XIGA provides the possibility of modelling the crack independently of the mesh and within a geometry presented exactly. Uncommonly, in this study T-splines are adopted in XIGA using analysis-suitable T-splines to approximate the displacement in any point $\zeta=\left(x_{1}, x_{2}\right)$ as follows

$$
\begin{aligned}
\boldsymbol{u}(\zeta) & =\sum_{i=1}^{n_{s}} R_{i}(\zeta) u_{i}+\sum_{j=1}^{n_{c f}} R_{j}(\zeta) H(\zeta) a_{j}+ \\
& +\sum_{k=1}^{n_{c t}} R_{k}(\zeta)\left(\sum_{l=1}^{4} F_{l}(\zeta) b_{k}^{l}\right)
\end{aligned}
$$

where $R$ is the analysis-suitable T-spline basis function Eq. (2), $H$ is the Heaviside function used to represent crack discontinuity, it equals 1 and -1 above and below the crack, respectively. $F_{l}$ are the asymptotic enrichment functions used to reproduce the singular field near the crack tips. $u, a$ and $b$ are vectors of degrees of freedom corresponding to classical $\left(n_{s}\right)$, crack face $\left(n_{c f}\right)$ and crack tip $\left(n_{c t}\right)$ control points, respectively.

For isotropic domains, the crack-tip enrichment function is defined by four components in each point as:

$$
\begin{aligned}
& \left\{F_{l}(r, \theta)\right\}_{l=1}^{4}= \\
& =\left\{\sqrt{r} \sin \frac{\theta}{2}, \sqrt{r} \cos \frac{\theta}{2}, \sqrt{r} \sin \frac{\theta}{2} \sin \theta, \sqrt{r} \cos \frac{\theta}{2} \sin \theta\right\},
\end{aligned}
$$

where $(r, \theta)$ are local crack tip polar coordinates.

XIGA discretization uses the same XFEM procedure to discrete Eq. (13)

$$
K \boldsymbol{u}=\boldsymbol{f},
$$

where $\boldsymbol{K}$ is the global stiffness matrix, $\boldsymbol{f}$ is applied forces vector and $\boldsymbol{u}$ is the displacement vector. For one finite element, they are defined as:

$$
\begin{aligned}
& {\left[K_{i j}\right]=\left[\begin{array}{lll}
{\left[k_{i j}{ }^{u u}\right]} & {\left[k_{i j}{ }^{a a}\right]} & {\left[k_{i j}{ }^{u b}\right]} \\
{\left[k_{i j}{ }^{a u}\right]} & {\left[k_{i j}^{a a}\right]} & {\left[k_{i j}{ }^{a b}\right]} \\
{\left[k_{i j}{ }^{b u}\right]} & {\left[k_{i j}{ }^{b a}\right]} & {\left[k_{i j}{ }^{b b}\right]}
\end{array}\right] ;} \\
& \boldsymbol{u}=\{u, a, b\}^{T} ; \\
& \boldsymbol{f}_{i}=\left\{f_{i}^{u}, f_{i}^{a}, f_{i}^{b}\right\}^{T},
\end{aligned}
$$

where

$$
\left[k_{i j}^{r s}\right]=\int_{\Omega_{e}}\left[\boldsymbol{B}_{i}^{r}\right]^{T}[C]\left[\boldsymbol{B}_{j}^{s}\right] d \Omega ; \quad r, s=u, a, b
$$

and

$$
\begin{gathered}
f_{i}^{u}=\int_{\Gamma_{t}^{e}} R_{i} f^{t} d \Gamma+\int_{\Omega^{e}} R_{i} f^{b} d \Omega \\
f_{i}^{a}=\int_{\Gamma_{t}^{e}} R_{i} H f^{t} d \Gamma+\int_{\Omega^{e}} R_{i} H f^{b} d \Omega ; \\
f_{i}^{b}=\int_{\Gamma_{t}^{e}} R_{i} F_{l} f^{t} d \Gamma+\int_{\Omega^{e}} R_{i} F_{l} f^{b} d \Omega \quad(l=1, \ldots, 4) .
\end{gathered}
$$

$\boldsymbol{C}$ is the matrix of elastic constants and $\boldsymbol{B}$ is the matrix of shape function derivatives which is given by: 


$$
\begin{aligned}
\boldsymbol{B}_{i}^{u} & =\left[\begin{array}{cc}
R_{i, x_{1}} & 0 \\
0 & R_{i, x_{2}} \\
R_{i, x_{2}} & R_{i, x_{1}}
\end{array}\right] ; \\
\boldsymbol{B}_{i}^{a} & =\left[\begin{array}{cc}
\left(R_{i} H\right)_{, x_{1}} & 0 \\
0 & \left(R_{i} H\right)_{, x_{2}} \\
\left(R_{i} H\right)_{, x_{2}} & \left(R_{i} H\right)_{, x_{1}}
\end{array}\right] ; \\
\boldsymbol{B}_{i}^{b} & =\left[\begin{array}{cc}
\left(R_{i} F_{l}\right)_{, x_{1}} & 0 \\
0 & \left(R_{i} F_{l}\right)_{, x_{2}} \\
\left(R_{i} F_{l}\right)_{, x_{2}} & \left(R_{i} F_{l}\right)_{, x_{1}}
\end{array}\right](l=1, \ldots, 4) .
\end{aligned}
$$

\section{Stress intensity factor computation}

For approximate this parameter we used the interaction Integral, is based on the definition of an auxiliary state to extract mixed mode stress intensity factors (SIF) as following:

$$
J=J^{a c t}+J^{a u x}+M,
$$

where $J^{a c t}$ and $J^{a u x}$ are the actual and auxiliary states $J$ integrals, and $J$ is the $J$ integral value for the superposition state, her general form is written as [21]:

$$
J=\int_{\ell}\left(\kappa \delta_{1 j}-\sigma_{i j} \frac{\partial u_{i}}{\partial x_{1}}\right) n_{j} d \ell
$$

and $M$ is the interaction integral defined as

$$
\begin{aligned}
M & =\int_{A}\left[\sigma_{i j} \frac{\partial u_{i}^{a u x}}{\partial x_{1}}+\sigma_{i j}^{a u x} \frac{\partial u_{i}}{\partial x_{1}}-\kappa \delta_{1 j}\right] \frac{\partial g}{\partial x_{i}} d \ell= \\
& =\frac{2}{E^{\prime}}\left(K_{I} K_{I}^{a u x}+K_{I I} K_{I I}^{a u x}\right),
\end{aligned}
$$

where $\kappa$ is the strain energy density, $\ell$ is an arbitrary contour surrounding the crack tip, $n_{j}$ is the $j$ th component of the outward unit normal to $\ell, \delta_{1 j}$ is the Kronecker delta, $g$ is a smoothly function varies from 1 on the crack-tip to 0 on the edge of the contour $\ell$, and $A$ is the area inside $\ell$. $E^{\prime}=E$ in plane stress and $E^{\prime}=E /\left(1-v^{2}\right)$ in plane strain ( $E$ is Young's modulus and $v$ is Poisson's ratio). The mode I and II stress intensity factors can be obtained as follows:

$$
K=\frac{E^{\prime}}{2} M,
$$

where state 1 and state 2 defined as $\left(K_{I}{ }^{a u x}=1, K_{I I}{ }^{a u x}=0\right)$ and $\left(K_{I}^{a u x}=0, K_{I I}^{a u x}=1\right)$, respectively.

\section{Numerical examples}

In this section, two numerical examples are simulated by programming all the above techniques and methods into one code. The geometric models of the below examples are constructed using cubic order in both directions. First, a rectangular plate with double edge cracks is chosen in order to study the convergence and the domain independence in the computations of SIF, and then a disk with center crack is described exactly and analyzed for different crack lengths and angles in order to verify the accuracy of the proposed approach. Four types of finite elements are distinguished in these examples according to their positions with the crack, the standard element contains $3 \times 3$ Gauss points, the element that having tip enriched control points contains $5 \times 5$ Gauss points and the sub-triangle technique is used for the tip-element by 7 Gauss points in each triangle, however the split element contains $6 \times 6$ Gauss points for the double edge crack problem and the sub-triangle technique is used by 7 Gauss points in each triangle for the cracked disk problem.

\subsection{Double edge cracked specimen under uniaxial tension}

Consider a tensile plate of width $2 w=10$ and height $2 h=20$ with double edge cracks of length $a=3$, as shown in Fig. 4. The analytical normalized SIF solution of this problem is given by:

$$
\bar{K}_{I}=\frac{K_{I}}{\sigma \sqrt{\pi a}}=T_{I}(a / w),
$$

where $T_{I}(a / \mathrm{w})$ is the analytical formula corresponding to the mode $I$, which can be computed as [22]

$$
T_{I}=1.12+0.203(a / w)-1.197(a / w)^{2}+1.93(a / w)^{3} .
$$

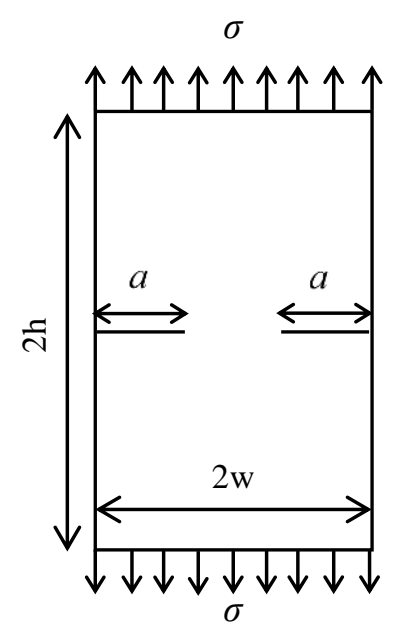

Fig. 4 Finite rectangular plate with double edge cracks

Different mesh configurations consist of 119, 251, 503, 655 and 1021 elements (all shown in Fig. 5) are used to study the convergence of the proposed approach with normalized $\mathrm{M}$-integral radius equal to 1 . Table 1 shows the obtained values with their errors, and Table 2 compares the results for different domain radius to study the domain independence in the case of 503 elements (Fig. 5, c).

According to Table 1, analysis suitable T-splines give us precise results for different numbers of control points and that attributed to the local refinement property. We can observe from Table 2 that the SIF values are almost not sensitive at all to the radius of the M-integral. 


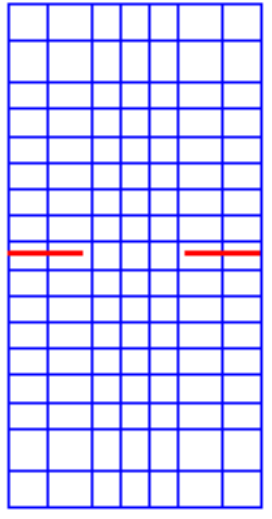

a

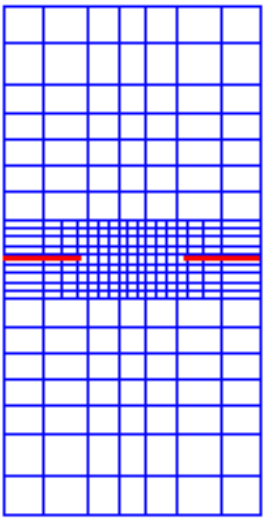

b

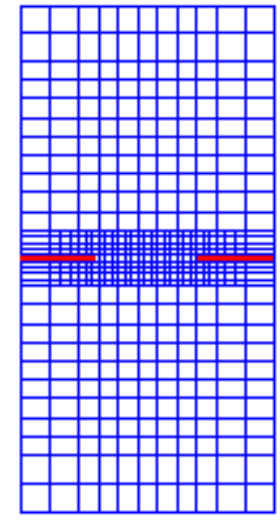

c

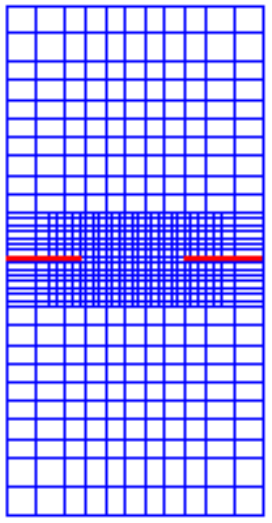

d

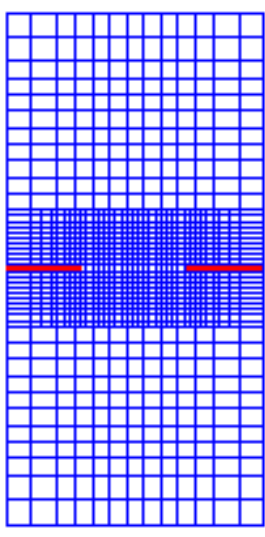

e

Fig. 5 Different mesh configurations used in the convergence study: a - 119 elements; b - 251 elements; c - 503 elements; d - 655 elements; e - 1021 elements

Table 1

Convergence of the SIF for various control nets

\begin{tabular}{|c|c|c|}
\hline Mesh & $\bar{K}_{I}$ & $\mid$ Error $\mid \%$ \\
\hline 119 & 1.0627 & 13.4468 \\
\hline 251 & 1.2390 & 0.9122 \\
\hline 503 & 1.2390 & 0.9122 \\
\hline 655 & 1.2385 & 0.8715 \\
\hline 1021 & 1.2361 & 0.6760 \\
\hline
\end{tabular}

Domain independence study

\begin{tabular}{|c|c|c|}
\hline Raduis & $\bar{K}_{I}$ & $\mid$ Error $\%$ \\
\hline 0.5 & 1.2426 & 1.2054 \\
\hline 0.7 & 1.2396 & 0.9611 \\
\hline 0.9 & 1.2413 & 1.0995 \\
\hline 1.1 & 1.2389 & 0.9041 \\
\hline
\end{tabular}

5.2. Disk with an inclined central crack subjected to compression load (Brazilian disk)

A disk with an inclined central crack subjected to compression load is considered for both fracture modes, as depicted in Fig. 6. Different inclined angles are tested, namely, $\varphi=0^{\circ}, 15^{\circ}, 30^{\circ}, 45^{\circ}, 60^{\circ}, 75^{\circ}$ and $90^{\circ}$ for different crack lengths. The analytical SIFs of this problem can be obtained by the following equations:

$$
\begin{aligned}
& K_{I}=T_{I}(\gamma) \sigma^{*} \sqrt{\pi a} ; \\
& K_{I I}=T_{I I}(\gamma) \sigma^{*} \sqrt{\pi a},
\end{aligned}
$$

where $\sigma^{*}$ is the characteristic stress $\left(\sigma^{*}=P_{0} /(\pi D t)\right), \gamma$ is crack length ratio $(\gamma=a / D), t$ is disk thickness, $T_{I}$ and $T_{I I}$ are the geometric functions corresponding to the mode I and II, respectively (all the geometric functions are taken from [23]).

In order to evaluate the mixed mode stress intensity factors in the case of $a=30 \mathrm{~mm}$, we used a mesh consists of 1337 control points and 1216 elements, as shown in Fig. 7, a. The crack tip and the crack face enriched control points are defined like in Fig. 8. The obtained results are compared with those of the reference [23] in Fig. 9. For different crack length ratios we used a uniform mesh (see Fig. 7, b) to show the variations of $K_{I}$ and $K_{I I}$ in Fig. 10.

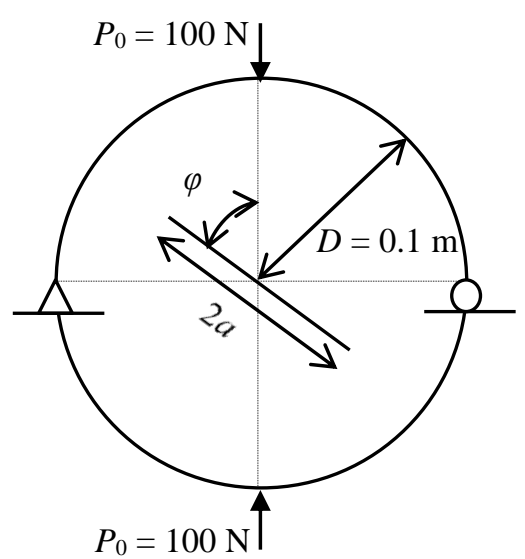

Fig. 6 Circular plate with central crack

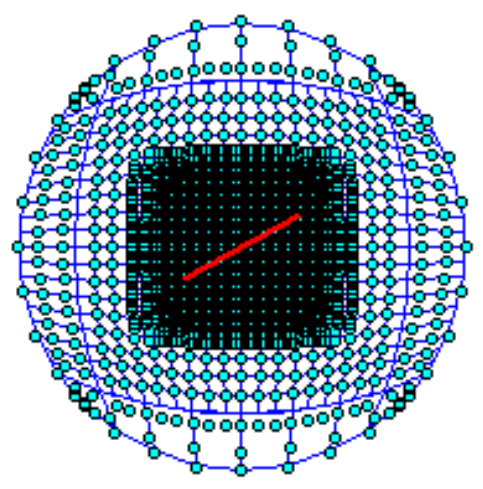

a

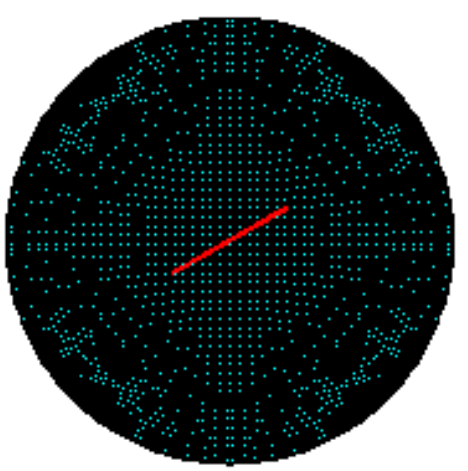

$\mathrm{b}$

Fig. 7 Control net with finite element mesh of circular plate using: a-T-spline (1216 elements); b - NURBS (4096 elements) 


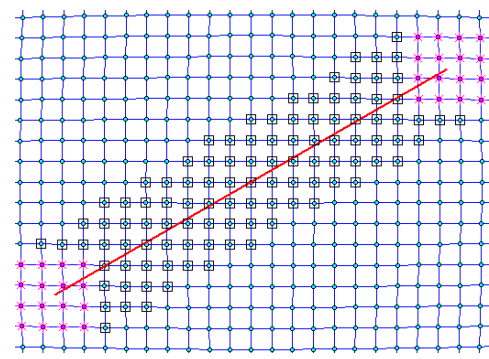

Fig. $8\left(^{*}\right)$ crack tip and ( $\left.\square\right)$ crack face enriched points of central cracked disk

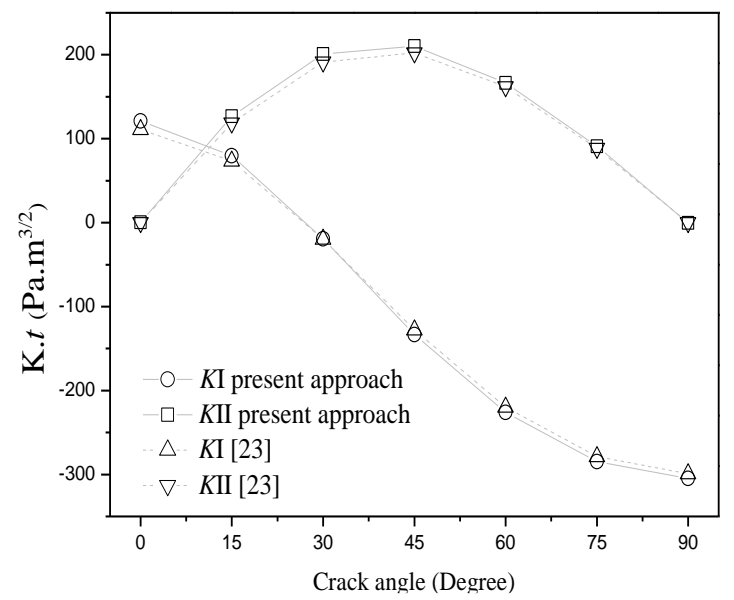

Fig. 9 Reference values and obtained values of SIF for different crack inclinations

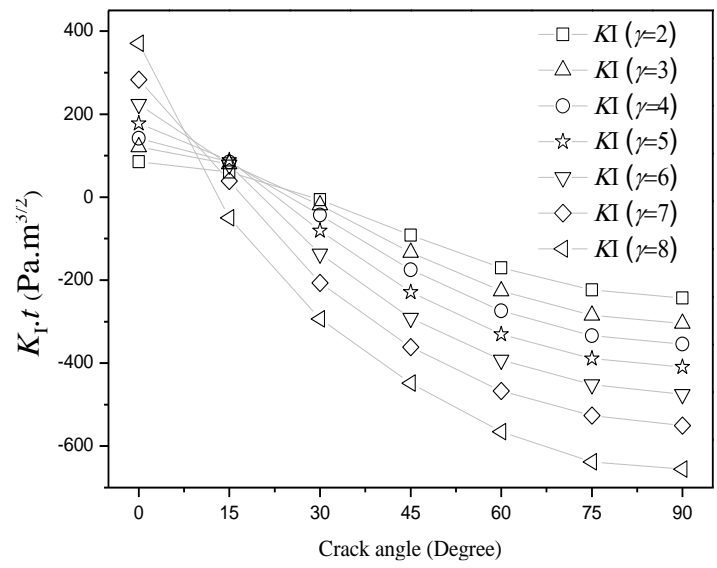

a

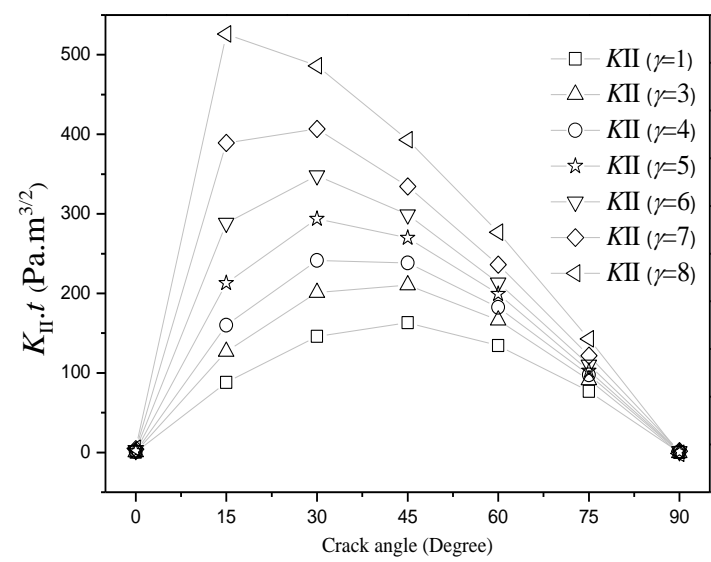

$\mathrm{b}$

Fig. 10 Variation of the obtained SIF with respect to different crack angles: a - mode I SIF; b - mode II SIF
According to Fig. 9, the results obtained from the present approach are in good agreement with the analytical results and that probably due to the ability of the T-splines to describe such geometries exactly. The mode I stress intensity factor reduces steadily with an increase in crack angle (Fig. 10, a), whereas the mode II stress intensity factor increases and reaches its maximum value at $\left(\varphi=45^{\circ}\right.$ for $\gamma=0.2,0.3$ and $\varphi=30^{\circ}$ for $\gamma=0.4,0.5,0.6,0.7$ and $\varphi=15^{\circ}$ for $\gamma=0.8$ ), and then decreases (Fig. 10, b. Therefore the crack angle of the maximum value of the mode II stress intensity factor depends on the crack length ratio.

\section{Conclusion}

In the present study, extended isogeometric analysis has been enhanced by analysis-suitable T-spline for modeling and analyzing cracks in plane problems. A compatible refinement algorithm has been contributed to make the local refinement and avoid the emergence of superfluous control points. The obtained stress intensity factors have been compared with analytical solutions, a good agreement proved the accuracy and efficiency of the proposed method.

\section{References}

1. Moës, N.M.; Dolbow, J.T.; Belytschko, T. 1999. A finite element method for crack growth without remeshing, International Journal for Numerical Methods in Engineering 46(1): 131-150.

http://dx.doi.org/10.1002/(SICI)1097-0207(19990910) 46:1<131::AID-NME726>3.0.CO;2-J.

2. Blandford, G.E.; Ingraffea, A.R.; Liggett. J.A. 1981. Two-dimensional stress intensity factor computations using the boundary element method, International Journal for Numerical Methods in Engineering 17(3): 387-404

http://dx.doi.org/10.1002/nme.1620170308.

3. Fleming, M.; Chu, Y.A.; Moran, B.; Belytschko, T.; Lu, Y.Y.; Gu, L. 1997. Enriched element-free Galerkin methods for crack tip fields, International journal for numerical methods in engineering 40(8): 14831504

http://dx.doi.org/10.1002/(SICI)1097-0207(19970430) 40:8<1483::AID-NME123>3.0.CO;2-6.

4. Ma, G.W.; An, X.M.; Zhang, H.H.; Li, L.X. 2009. Modeling complex crack problems using the numerical manifold method, International Journal of Fracture 156(1): 21-35.

http://dx.doi.org/10.1007/s10704-009-9342-7.

5. Ferretti, E. 2003. Crack propagation modeling by remeshing using the Cell Method (CM), CMES- Computer Modeling in Engineering and Sciences 4(1): 5172.

http://dx.doi.org/10.3970/cmes.2003.004.051.

6. Hughes, T.J.; Cottrell, J.A.; Bazilevs, Y. 2005. Isogeometric analysis: CAD, finite elements, NURBS, exact geometry and mesh refinement, Computer Methods in Applied Mechanics and Engineering 194(39): 41354195. http://dx.doi.org/10.1016/j.cma.2004.10.008.

7. Auricchio, F.; da Veiga, L.B.; Hughes, T.J.R.; Reali, A.; Sangalli, G. 2012. Isogeometric collocation for elastostatics and explicit dynamics, Computer Methods 
in Applied Mechanics and Engineering 249: 2-14. http://dx.doi.org/10.1016/j.cma.2012.03.026.

8. Thai, C.H.; Ferreira, A.J.M.; Bordas, S.P.A.; Rabczuk, T.; Nguyen-Xuan, H. 2014. Isogeometric analysis of laminated composite and sandwich plates using a new inverse trigonometric shear deformation theory, European Journal of Mechanics-A/Solids 43: 89-108.

http://dx.doi.org/10.1016/j.euromechsol.2013.09.001.

9. Bazilevs, Y.; Calo, V.M.; Hughes, T.J.R.; Zhang, Y. 2008. Isogeometric fluid-structure interaction: theory, algorithms, and computations, Computational Mechanics 43(1): 3-37. http://dx.doi.org/10.1007/s00466-008-0315-x.

10. Buffa, A.; Sangalli, G.; Vázquez, R. 2014. Isogeometric methods for computational electromagnetics: Bspline and T-spline discretizations, Journal of Computational Physics 257: 1291-1320. http://dx.doi.org/10.1016/j.jcp.2013.08.015.

11. De Lorenzis, L.; Temizer, I.; Wriggers, P.; Zavarise, G. 2011. A large deformation frictional contact formulation using NURBS-based isogeometric analysis, International Journal for Numerical Methods in Engineering 87(13): 1278-1300. http://dx.doi.org/10.1002/nme.3159.

12. Chang, K.; Hughes, T.J.R.; Calo, V.M. 2012. Isogeometric variational multiscale large-eddy simulation of fully-developed turbulent flow over a wavy wall, Computers \& Fluids 68: 94-104. http://dx.doi.org/10.1016/j.compfluid.2012.06.009.

13. Hsu, M.C.; Akkerman, I.; Bazilevs, Y. 2011. Highperformance computing of wind turbine aerodynamics using isogeometric analysis, Computers \& Fluids 49(1): 93-100.

http://dx.doi.org/10.1016/j.compfluid.2011.05.002.

14. Temizer, I. 2014. Multiscale thermomechanical contact: Computational homogenization with isogeometric analysis, International Journal for Numerical Methods in Engineering 97(8): 582-607.

http://dx.doi.org/10.1002/nme.4604.

15. Benson, D.J.; Bazilevs, Y.; De Luycker, E.; Hsu, M.C.; Scott, M.; Hughes, T.J.R.; Belytschko, T. 2010. A generalized finite element formulation for arbitrary basis functions: from isogeometric analysis to XFEM, International Journal for Numerical Methods in Engineering 83(6): 765-785. http://dx.doi.org/10.1002/nme.2864.

16. De Luycker, E.; Benson, D.J.; Belytschko, T.; Bazilevs, Y.; Hsu, M.C. 2011. X-FEM in isogeometric analysis for linear fracture mechanics, International Journal for Numerical Methods in Engineering 87(6): 541-565.

http://dx.doi.org/10.1002/nme.3121.

17.Sederberg, T.W.; Zheng, J.; Bakenov, A.; Nasri, A. 2003. T-splines and T-NURCCs, In ACM Transactions on Graphics 22(3): 477-484.

http://dx.doi.org/10.1145/882262.882295.

18.Sederberg, T.W.; Cardon, D.L.; Finnigan, G.T.; North, N.S.; Zheng, J.; Lyche, T. 2004. T-spline sim- plification and local refinement, In ACM Transactions on Graphics (TOG) 23(3): 276-283.

http://dx.doi.org/10.1145/1015706.1015715.

19. Li, X.; Zheng, J.; Sederberg, T.W.; Hughes, T.J.; Scott, M.A. 2012. On linear independence of T-spline blending functions, Computer Aided Geometric Design 29(1): 63-76.

http://dx.doi.org/10.1016/j.cagd.2011.08.005.

20. Scott, M.A.; Li, X.; Sederberg, T.W.; Hughes, T.J. R. 2012. Local refinement of analysis-suitable Tsplines, Computer Methods in Applied Mechanics and Engineering 213: 206-222. http://dx.doi.org/10.1016/j.cma.2011.11.022.

21. Rice, J.R. 1968. A path independent integral and the approximate analysis of strain concentration by notches and cracks, Journal of Applied Mechanics 35(2): 379386. http://dx.doi.org/10.1115/1.3601206.

22. Tada, H.; Paris, P.C.; Irwin, G.R. 2000. The Stress Analysis of Cracks Handbook, New York: ASME Press, 696 p. http://dx.doi.org/10.1115/1.801535.

23. Fett, T. 2001. Stress intensity factors and T-stress for internally cracked circular disks under various boundary conditions, Engineering Fracture Mechanics 68(9): 1119-1136.

http://dx.doi.org/10.1016/S0013-7944(01)00025-X.

\section{S. H. Habib, I. Belaidi}

\section{EXTENDED ISOGEPMETRIC ANALYSIS USING ANALYSIS-SUITABLE T-SPLINES FOR PLANE CRACK PROBLEMS}

S u m m a r y

This study is dedicated to modeling cracks in plane problems by applying the recent technique analysissuitable T-splines in the extended isogeometric analysis. A new local refinement algorithm is integrated for increasing the solution accuracy and reducing the excessive propagation of control points. However the singular fields near a crack tip are reproduced by the crack tip enrichment functions, and the Heaviside function is used to represent crack discontinuity. The results accuracy is tested by evaluation the mixed mode stress intensity factors which are computed by means of the interaction integral approach (M integral). The obtained results are compared with the analytical methods.

Keywords: Extended isogeometric analysis, analysissuitable T-splines, local refinement, M-integral, isotropic materials.

Received October 23, 2015

Accepted February 06, 2017 